\title{
Quantum Random Walk Approximation on Locally Compact Quantum Groups
}

\author{
J. MARTIN LINDSAY ${ }^{1}$ and ADAM G. SKALSKI ${ }^{2}$ \\ ${ }^{1}$ Department of Mathematics and Statistics, Lancaster University, Lancaster LA1 $4 Y F$, \\ UK.e-mail: j.m.lindsay@lancaster.ac.uk \\ ${ }^{2}$ Institute of Mathematics of the Polish Academy of Sciences, ul.Śniadeckich 8, \\ 00-956 Warsaw, Poland. e-mail: a.skalski@impan.pl
}

Received: 27 April 2012 / Revised: 14 January 2013 / Accepted: 26 January 2013

Published online: 17 February 2013 - (C) The Author(s) 2013. This article is published with open access at Springerlink.com

\begin{abstract}
A natural scheme is established for the approximation of quantum Lévy processes on locally compact quantum groups by quantum random walks. We work in the somewhat broader context of discrete approximations of completely positive quantum stochastic convolution cocycles on $C^{*}$-bialgebras.
\end{abstract}

Mathematics Subject Classification (2000). Primary 46L53, 81S25; Secondary 22A30, 47L25, 16W30.

Keywords. quantum random walk, quantum Lévy process, noncommutative probability, locally compact quantum group, $C^{*}$-bialgebra, stochastic cocycle.

\section{Introduction}

In [19] we developed a theory of quantum stochastic convolution cocycles on counital multiplier $C^{*}$-bialgebras, extending the algebraic theory of quantum Lévy processes created by Schürmann and co-workers (see [25] and references therein, and, for a simplified treatment [17]), and the topological theory of quantum stochastic convolution cocycles on compact quantum groups and operator space coalgebras developed by the authors [18]. Here we apply the results of [19] to introduce and analyse a straightforward scheme for the approximation of such cocycles by quantum random walks. In particular we obtain results on Markovregular quantum Lévy processes on locally compact quantum semigroups, extending and strengthening results in [11] for the compact case. Our analysis exploits a recent approximation theorem of Belton [6], which extends that of [24] (used in [11]). The approximation scheme closely mirrors the way in which Picard iteration operates in the construction of solutions of quantum stochastic differential equations [15].

The study of quantum random walks on quantum groups was initiated by Biane in the early 1990s (starting with [7]). Some combinatorial, probabilistic and physical 
interpretations can be found in Chapter 5 of [21]. Recent work has concentrated on discrete quantum groups and the development of (Poisson and Martin) boundary theory for quantum random walks (see [22] and references therein). Random walks of the type considered here and in [11] are discussed in [10] in the context of finite quantum groups. For standard quantum stochastic cocycles on operator algebras, operator spaces and Hilbert spaces (see [15], and references therein), quantum random walk approximation $[6,12,16,24]$ has seen recent applications in the probability theory and mathematical physics literature (e.g. $[2,8])$.

\section{Preliminaries}

In this section we briefly recall some definitions and relevant facts about strict maps and their extensions, matrix spaces over an operator space, structure maps with respect to a character on a $C^{*}$-algebra, multiplier $C^{*}$-bialgebras and quantum stochastic convolution cocycles; we refer to [19] for a detailed account.

General notations. The multiplier algebra of a $C^{*}$-algebra $\mathrm{A}$ is denoted by $M(\mathrm{~A})$ (in [19] the notation $\widetilde{A}$ was used). The symbols $\otimes, \otimes$ and $\bar{\otimes}$ are used, respectively, for linear/algebraic, spatial/minimal and ultraweak, tensor products of spaces, and also, respectively, for linear, completely bounded and ultraweakly continuous completely bounded, tensor products of maps (see e.g. [9]). For a subset $S$ of a vector space $V$, its linear span is denoted $\operatorname{Lin} S$. The following notations are used for vector functionals, bra-/-ket operators, ampliations and augmented spaces:

$$
\begin{aligned}
& \omega_{\xi}: B(\mathrm{~h}) \rightarrow \mathbb{C}, A \mapsto\langle\xi, A \xi\rangle, \quad|\xi\rangle: \mathbb{C} \rightarrow \mathrm{h}, \lambda \mapsto \lambda \xi, \quad\langle\xi|:=| \xi\rangle^{*} \quad(\xi \in \mathrm{h}), \\
& |\mathrm{h}\rangle:=\{|\xi\rangle: \xi \in \mathrm{h}\}=B(\mathbb{C} ; \mathrm{h}), \quad \iota_{\mathrm{h}}: B\left(\mathrm{H} ; \mathrm{H}^{\prime}\right) \rightarrow B\left(\mathrm{H} \otimes \mathrm{h} ; \mathrm{H}^{\prime} \otimes \mathrm{h}\right), T \mapsto T \otimes I_{\mathrm{h}}, \\
& \widehat{\mathrm{K}}:=\mathbb{C} \oplus \mathrm{K}, \quad \widehat{c}:=\left(\begin{array}{l}
1 \\
c
\end{array}\right) \quad \text { for } c \in \mathrm{K} \quad \text { and } \quad \Delta^{\mathrm{QS}}:=P_{\{0\} \oplus \mathrm{K}}=\left[\begin{array}{cc}
0 & \\
& I_{\mathrm{K}}
\end{array}\right] \in B(\widehat{\mathrm{K}}) .
\end{aligned}
$$

Here $\mathrm{h}$ is a Hilbert space and context determines the Hilbert spaces $\mathrm{H}, \mathrm{H}^{\prime}$ and (for $\Delta^{\mathrm{QS}}$ ) also $\mathrm{K}$; the superscript QS is there to avoid confusion with coproducts.

\subsection{STRICT MAPS AND THEIR EXTENSIONS}

If $\mathrm{A}_{1}, \mathrm{~A}_{2}$ are $C^{*}$-algebras then a map $\varphi: \mathrm{A}_{1} \rightarrow M\left(\mathrm{~A}_{2}\right)$ is called strict if it is bounded and continuous in the strict topology on bounded subsets. The space of all such maps is denoted $B_{\beta}\left(\mathrm{A}_{1} ; M\left(\mathrm{~A}_{2}\right)\right)$. Each map $\varphi \in B_{\beta}\left(\mathrm{A}_{1} ; M\left(\mathrm{~A}_{2}\right)\right)$ has a unique strict extension $\widetilde{\varphi}: M\left(\mathrm{~A}_{1}\right) \rightarrow M\left(\mathrm{~A}_{2}\right)$. This allows the following natural composition operation: if $\psi \in B_{\beta}\left(\mathrm{A}_{2} ; M\left(\mathrm{~A}_{3}\right)\right)$ for another $C^{*}$-algebra $\mathrm{A}_{3}$ then $\psi \circ \varphi$ is defined to be $\widetilde{\psi} \circ \varphi$. A map $\varphi \in B_{\beta}\left(\mathrm{A}_{1} ; M\left(\mathrm{~A}_{2}\right)\right)$ is called preunital if its strict extension is unital. Thus, for $*$-homomorphic maps, preunital is equivalent to nondegenerate [14].

Every completely bounded map from a $C^{*}$-algebra to the algebra of all bounded operators on a Hilbert space is automatically strict, when the latter is viewed as the multiplier algebra of the algebra of compact operators (see [19, Section 2]). 


\subsection{MATRIX SPACES}

For an operator space $\mathrm{V}$ in $B(\mathrm{H} ; \mathrm{K})$ and full operator space $B=B(\mathrm{~h} ; \mathrm{k})$, the $(\mathrm{h}, \mathrm{k})$ matrix space over $\mathrm{V}$, denoted $\mathrm{V} \otimes_{\mathrm{M}} B$, is the following operator space:

$$
\left\{A \in B(\mathrm{H} \otimes \mathrm{h}, \mathrm{K} \otimes \mathrm{k}): \forall_{\omega \in B_{*}}\left(\mathrm{id}_{B(\mathrm{H} ; \mathrm{K})} \bar{\otimes} \omega\right)(A) \in \mathrm{V}\right\} .
$$

It lies between $\mathrm{V} \otimes B$ and $\overline{\mathrm{V}}^{\mathrm{uw}} \bar{\otimes} B$ and is equal to the latter when $\mathrm{V}$ is ultraweakly closed. For any map $\varphi \in C B\left(\mathrm{~V}_{1} ; \mathrm{V}_{2}\right)$, between operator spaces, the map $\varphi \otimes \operatorname{id}_{B}$ extends uniquely to a completely bounded $\operatorname{map} \varphi \otimes_{\mathrm{M}} \operatorname{id}_{B}: \mathrm{V}_{1} \otimes_{\mathrm{M}} B \rightarrow \mathrm{V}_{2} \otimes_{\mathrm{M}} B$ [20]. This construction is compatible with strict tensor products and strict extension, as is shown in Section 1 of [19].

\section{3. $\chi$-STRUCTURE MAPS}

Let $(\mathrm{A}, \chi)$ be a $C^{*}$-algebra with character. A $\chi$-structure map is a linear map $\varphi$ : $A \rightarrow B(\widehat{h})$, for some Hilbert space $h$, satisfying

$$
\varphi\left(a^{*} b\right)=\varphi(a)^{*} \chi(b)+\chi(a)^{*} \varphi(b)+\varphi(a)^{*} \Delta^{\mathrm{QS}} \varphi(b),
$$

in which $\Delta^{\mathrm{QS}}$ is given by (1.1). The following automatic implementability result is key ([18, Theorem A6] and [19]).

THEOREM 1.1. Let $(\mathrm{A}, \chi)$ be a $C^{*}$-algebra with character and let $\varphi$ be a linear map $\mathrm{A} \rightarrow B(\widehat{\mathrm{h}})$, for some Hilbert space $\mathrm{h}$. Then the following are equivalent:

(i) $\varphi$ is a $\chi$-structure map.

(ii) $\varphi$ is implemented by a pair $(\pi, \xi)$ consisting of a ${ }^{*}$-homomorphism $\pi: \mathrm{A} \rightarrow B(\mathrm{~h})$ and vector $\xi \in \mathrm{h}$, that is $\varphi$ has block matrix form

$$
Z^{*} v(\cdot) Z \text { where } Z:=\left[\begin{array}{ll}
|\xi\rangle & I_{\mathrm{h}}
\end{array}\right] \text { and } v:=\pi-\iota_{\mathrm{h}} \circ \chi \text {. }
$$

Moreover, if $\varphi$ is a $\chi$-structure map with such a block matrix form then it is necessarily strict, and $\pi$ is nondegenerate if and only if $\widetilde{\varphi}(1)=0$.

\subsection{MULTIPLIER $C^{*}$ - BIALGEBRAS}

A (multiplier) $C^{*}$-bialgebra is a $C^{*}$-algebra $\mathrm{B}$ with coproduct, that is a nondegenerate ${ }^{*}$-homomorphism $\Delta: \mathrm{B} \rightarrow M(\mathrm{~B} \otimes \mathrm{B})$ satisfying the coassociativity conditions

$$
\left(\operatorname{id}_{\mathrm{B}} \otimes \Delta\right) \circ \Delta=\left(\Delta \otimes \mathrm{id}_{\mathrm{B}}\right) \circ \Delta .
$$

A counit for $(B, \Delta)$ is a character $\epsilon$ on $B$ satisfying the counital property:

$$
\left(\mathrm{id}_{\mathrm{B}} \otimes \epsilon\right) \circ \Delta=\left(\epsilon \otimes \mathrm{id}_{\mathrm{B}}\right) \circ \Delta=\mathrm{id}_{\mathrm{B}} .
$$

Examples of counital $C^{*}$-bialgebras include all locally compact quantum groups in the universal setting [13] - in particular, the coamenable locally compact quantum groups [4]. 
Let $\mathrm{B}$ be a $C^{*}$-bialgebra. The convolute of maps $\phi_{1} \in \operatorname{Lin} C P_{\beta}\left(\mathrm{B} ; M\left(\mathrm{~A}_{1}\right)\right)$ and $\phi_{2} \in \operatorname{Lin} C P_{\beta}\left(\mathrm{B} ; M\left(\mathrm{~A}_{2}\right)\right)$, for $C^{*}$-algebras $\mathrm{A}_{1}$ and $\mathrm{A}_{2}$, is defined as the following composition of strict maps:

$$
\phi_{1} \star \phi_{2}=\left(\phi_{1} \otimes \phi_{2}\right) \circ \Delta \in \operatorname{Lin} C P_{\beta}\left(\mathrm{B} ; M\left(\mathrm{~A}_{1} \otimes \mathrm{A}_{2}\right)\right) ;
$$

the same notation is used for its strict extension. The convolution operation is easily seen to be associative. Moreover, by automatic strictness, and the decomposability property:

$C B(\mathrm{~B} ; B(\mathrm{~h}))=\operatorname{Lin} C P(\mathrm{~B} ; B(\mathrm{~h})), \quad$ for any Hilbert space $\mathrm{h}$;

it follows that any maps $\varphi_{1} \in C B\left(\mathrm{~B} ; B\left(\mathrm{~h}_{1}\right)\right)$ and $\varphi_{2} \in C B\left(\mathrm{~B} ; B\left(\mathrm{~h}_{2}\right)\right)$, for Hilbert spaces $h_{1}$ and $h_{2}$, may be convolved:

$$
\varphi_{1} \star \varphi_{2} \in C B\left(\mathrm{~B} ; B\left(\mathrm{~h}_{1} \otimes \mathrm{h}_{2}\right)\right) .
$$

\subsection{QUANTUM STOCHASTIC CONVOLUTION COCYCLES}

We now fix, for the rest of the paper, a complex Hilbert space $\mathrm{k}$ referred to as the noise dimension space and a counital $C^{*}$-bialgebra $\mathrm{B}$.

For a subinterval $J$ of $\mathbb{R}_{+}$, let $\mathcal{F}_{J}$ denote the symmetric Fock space over $L^{2}(J ; \mathrm{k})$ and write $I_{J}$ for the identity operator on $\mathcal{F}_{J}$ and $\mathcal{F}$ for $\mathcal{F}_{[0, \infty[}$. Also let $\mathcal{E}$ denote the linear span of $\left\{\varepsilon(g): g \in L^{2}\left(\mathbb{R}_{+} ; \mathbf{k}\right)\right\}$, where $\varepsilon(g)$ denotes the exponential vector $\left((n !)^{-\frac{1}{2}} g^{\otimes n}\right)_{n \geq 0}$ in $\mathcal{F}$. Thus $\left\langle\varepsilon\left(f^{\prime}\right), \varepsilon(f)\right\rangle=\exp \left\langle f^{\prime}, f\right\rangle\left(f^{\prime}, f \in L^{2}(J ; \mathrm{k})\right)$ and, for $0 \leq r \leq t$, the natural unitary operator $\mathcal{F} \rightarrow \mathcal{F}_{[0, r[} \otimes \mathcal{F}_{[r, t[} \otimes \mathcal{F}_{[t, \infty[}$ is determined by the prescription $\varepsilon(g) \mapsto \varepsilon\left(\left.g\right|_{[0, r[}\right) \otimes \varepsilon\left(\left.g\right|_{[r, t]}\right) \otimes \varepsilon\left(\left.g\right|_{[t, \infty[}\right)\left(g \in L^{2}\left(\mathbb{R}_{+} ; \mathbf{k}\right)\right)[15,23]$.

For $\varphi \in C B(\mathrm{~B} ; B(\widehat{\mathrm{k}}))$, the coalgebraic QS differential equation

$$
\mathrm{d} l_{t}=l_{t} \star \mathrm{d} \Lambda_{\varphi}(t), \quad l_{0}=\iota \mathcal{F} \circ \epsilon,
$$

has a unique form solution, denoted $l^{\varphi}$; it is actually a strong solution. The process $l^{\varphi}$ is a QS convolution cocycle on B; moreover, conversely any Markov-regular, completely positive, contractive, QS convolution cocycle on B is of the form $l^{\varphi}$ for a unique $\varphi \in C B(\mathrm{~B} ; B(\widehat{\mathrm{k}}))$. For completely bounded processes the cocycle relation reads as follows (after some natural identifications are made):

$$
l_{s+t}=l_{s} \star\left(\sigma_{s} \circ l_{t}\right), \quad l_{0}=\iota \mathcal{F} \circ \epsilon, \quad s, t \in \mathbb{R}_{+},
$$

where $\left(\sigma_{s}\right)_{s \geq 0}$ is the semigroup of right shifts on $B(\mathcal{F})$. Markov regularity means that each of the associated convolution semigroups of the cocycle is norm continuous. In this situation, the map $\varphi$ is referred to as the stochastic generator of the QS convolution cocycle. A QS convolution cocycle $l$ is said to be completely positive, preunital, or *-homomorphic, if each $l_{t}$ has that property. The form of the generators of such cocycles is characterised in Theorem 5.2 of [19]. 


\section{Approximation by Discrete Evolutions}

We now show that any Markov-regular, completely positive, contractive QS convolution cocycle on B may be approximated in a strong sense by discrete completely positive evolutions, and that the discrete evolutions may be chosen to be *-homomorphic and/or preunital, if the cocycle is.

Belton's condition [6] for discrete approximation of standard Markov-regular QS cocycles [15] nicely translates to the convolution context using the techniques developed in [19]. We show this first. Denote by $\Xi_{n}^{(h)}(h>0, n \in \mathbb{N})$ the injective *-homomorphism

$$
B\left(\widehat{\mathrm{k}}^{\otimes n}\right)=B(\widehat{\mathrm{k}})^{\bar{\otimes} n} \rightarrow B\left(\mathcal{F}_{[0, h n[}\right) \otimes I_{[h n, \infty[}=\left(\bar{\bigotimes}_{j=1}^{n} B\left(\mathcal{F}_{[(j-1) h, j h[}\right)\right) \otimes I_{[h n, \infty[}
$$

arising from the discretisation of Fock space [2,5]. Thus

$$
\Xi_{n}^{(h)}: A \mapsto J_{n}^{(h)} A J_{n}^{(h) *} \otimes I_{[h n, \infty[}
$$

where

$$
\begin{aligned}
& J_{n}^{(h)}:=\bigotimes_{j=1}^{n} J_{n, j}^{(h)}, \quad \text { for the isometries } \\
& J_{n, j}^{(h)}: \widehat{\mathrm{k}} \mapsto \mathcal{F}_{[(j-1) h, j h[},\left(\begin{array}{l}
z \\
c
\end{array}\right) \mapsto\left(z, h^{-1 / 2} c_{[(j-1) h, j h[}, 0,0, \ldots\right) .
\end{aligned}
$$

Also write $\Xi_{n, \varepsilon}^{(h)}$ for the completely bounded map

$$
\Xi_{n}^{(h)}(\cdot)|\varepsilon\rangle: B\left(\widehat{\mathrm{k}}^{\otimes n}\right) \rightarrow|\mathcal{F}\rangle, \quad \text { where } h>0, n \in \mathbb{N} \text { and } \varepsilon \in \mathcal{E} .
$$

For a map $\Psi \in C B\left(\mathrm{~V} ; \mathrm{V} \otimes_{\mathrm{M}} B(\widehat{\mathrm{k}})\right)$, in which $\mathrm{V}$ is a concrete operator space, its composition iterates $\left(\Psi^{\bullet n}\right)_{n \in \mathbb{Z}_{+}}$are defined recursively by

$$
\Psi^{\bullet 0}:=\mathrm{id} \mathrm{V}, \quad \Psi^{\bullet n}:=\left(\Psi^{\bullet(n-1)} \otimes_{\mathrm{M}} \operatorname{id}_{B(\widehat{\mathrm{k}})}\right) \circ \Psi \in C B\left(\mathrm{~V} ; \mathrm{V} \otimes_{\mathrm{M}} B\left(\widehat{\mathrm{k}}^{\otimes n}\right)\right), \quad n \in \mathbb{N} .
$$

Similarly, for a map $\psi \in C B(\mathrm{~B} ; B(\widehat{\mathrm{k}}))$, its convolution iterates $\left(\psi^{\star n}\right)_{n \in \mathbb{Z}_{+}}$are defined by

$$
\psi^{\star 0}:=\epsilon, \quad \psi^{\star n}=\psi^{\star(n-1)} \star \psi \in C B\left(\mathrm{~B} ; B\left(\widehat{\mathrm{k}}^{\otimes n}\right)\right) \quad(n \in \mathbb{N}) .
$$

As usual we are viewing $B\left(\widehat{\mathrm{k}}^{\otimes n}\right)$ as the multiplier algebra of $K\left(\widehat{\mathrm{k}}^{\otimes n}\right)$ here, and invoking the remark containing (1.3), to ensure meaning for the above convolutions.

We need the following block matrix operators, on a Hilbert space of the form $\widehat{H}$ :

$$
\mathcal{S}_{h}:=\left[\begin{array}{cc}
h^{-1 / 2} & \\
& I_{\mathrm{H}}
\end{array}\right], \quad h>0,
$$

and write $\Sigma_{h}$ for the map $X \mapsto \mathcal{S}_{h} X \mathcal{S}_{h}$ on $B(\widehat{\mathrm{H}})$. Such conjugations provide the correct scaling for quantum random-walk approximation [16]. 
THEOREM 2.1. Let $\varphi \in C B(\mathrm{~B} ; B(\widehat{\mathrm{k}}))$. Suppose that there is a family of maps $\left(\psi^{(h)}\right)_{0<h<H}$ in $C B(\mathrm{~B} ; B(\widehat{\mathrm{k}}))$ for some $H>0$, satisfying

$$
\left\|\varphi-\Sigma_{h} \circ\left(\psi^{(h)}-\iota_{\widehat{\mathrm{k}}} \circ \epsilon\right)\right\|_{\mathrm{cb}} \rightarrow 0 \quad \text { as } h \rightarrow 0 .
$$

Then the convolution iterates $\left(\psi_{n}^{(h)}:=\left(\psi^{(h)}\right)^{\star n}\right)_{n \in \mathbb{Z}_{+}}$satisfy

$$
\sup _{t \in[0, T]}\left\|l_{t, \varepsilon}^{\varphi}-\Xi_{[t / h], \varepsilon}^{(h)} \circ \psi_{[t / h]}^{(h)}\right\|_{\mathrm{cb}} \rightarrow 0 \quad \text { as } h \rightarrow 0 \quad\left(T \in \mathbb{R}_{+}, \varepsilon \in \mathcal{E}\right),
$$

where $l_{t, \varepsilon}^{\varphi}:=l_{t}^{\varphi}(\cdot)|\varepsilon\rangle \in C B(\mathrm{~B} ;|\mathcal{F}\rangle)$ and $\Xi_{[t / h], \varepsilon}^{(h)}$ is given by $(2.1)$.

Proof. Denote the enveloping von Neumann algebra of $\mathrm{B}$ by $\overline{\mathrm{B}}$ and let $\phi$ and $\Psi^{(h)}$ in $C B_{\sigma}(\overline{\mathrm{B}} ; B(\widehat{\mathrm{k}}))$ and $\epsilon \in \overline{\mathrm{B}}_{*}$ denote, respectively, the normal extensions of $(\operatorname{id} \otimes \varphi) \circ \Delta$ and $\left(\operatorname{id} \otimes \psi^{(h)}\right) \circ \Delta$, and the counit of B. It follows from [19] (specifically, Proposition 2.1 and remarks after Theorem 1.2) that the maps transforming $\varphi$ into $\phi$ and $\psi^{(h)}$ into $\Psi^{(h)}$ are complete isometries. Therefore

$$
\left\|\left(\mathrm{id}_{\overline{\mathrm{B}}} \bar{\otimes} \Sigma_{h}\right) \circ\left(\Psi^{(h)}-\iota_{\widehat{\mathrm{k}}}\right)-\phi\right\|_{\mathrm{cb}}=\left\|\Sigma_{h} \circ\left(\psi^{(h)}-\iota_{\widehat{\mathrm{k}}} \circ \epsilon\right)-\varphi\right\|_{\mathrm{cb}},
$$

which tends to 0 as $h \rightarrow 0$ by assumption. Therefore, by Theorem 7.6 of [6],

$$
\sup _{t \in[0, T]}\left\|\left(\operatorname{id}_{\overline{\mathrm{B}}} \bar{\otimes} \Xi_{[t / h], \varepsilon}^{(h)}\right) \circ \Psi_{[t / h]}^{(h)}-k_{t, \varepsilon}^{\phi}\right\|_{\mathrm{cb}} \rightarrow 0 \quad \text { as } h \rightarrow 0,
$$

where $\Psi_{n}^{(h)}:=\left(\Psi^{(h)}\right)^{\bullet n}, k^{\phi}$ denotes the 'standard' QS cocycle generated by $\phi$, that is, the unique weakly regular weak solution of the QS differential equation

$$
\mathrm{d} k_{t}=k_{t} \circ \mathrm{d} \Lambda_{\phi}(t), \quad k_{0}=\iota \mathcal{F}
$$

and $k_{t, \varepsilon}^{\phi}:=k_{t}^{\phi}(\cdot) I \otimes|\varepsilon\rangle \in C B_{\sigma}(\overline{\mathrm{B}} ; \overline{\mathrm{B}} \bar{\otimes}|\mathcal{F}\rangle)$ (see [15]). It follows from Section 4 of [19] that $l_{t, \varepsilon}^{\varphi}=\left.\bar{l}_{t, \varepsilon}^{\varphi}\right|_{\mathrm{B}}$ where $\bar{l}_{t, \varepsilon}^{\varphi}=\left(\epsilon \bar{\otimes} \mathrm{id}_{|\mathcal{F}\rangle}\right) \circ k_{t, \varepsilon}^{\phi}\left(t \in \mathbb{R}_{+}, \varepsilon \in \mathcal{E}\right)$. The result therefore follows from the easily checked identity $\left.(\epsilon \bar{\otimes} \mathrm{id}) \circ \Psi_{n}^{(h)}\right|_{\mathrm{B}}=\psi_{n}^{(h)}\left(n \in \mathbb{Z}_{+}\right)$.

Remark. Multiplicativity of the coproduct is not used in the above proof; the proper hypothesis on B is that it be a multiplier $C^{*}$-hyperbialgebra (see [19, Section 2]).

For the next two propositions coproducts play no role.

PROPOSITION 2.2. Let $(\mathrm{A}, \chi)$ be a $C^{*}$-algebra with character and let $\varphi: \mathrm{A} \rightarrow B(\widehat{\mathrm{h}})$ be a $\chi$-structure map. Letting $(\pi, \xi)$ be an implementing pair for $\varphi$ (in the sense of Theorem 1.1), set $h(\xi)$ equal to $\|\xi\|^{-2}$ (or $\infty$ if $\left.\xi=0\right)$ and, for $0<h<h(\xi)$, define

$$
U_{\xi}^{(h)}:=\left[\begin{array}{cc}
c_{h, \xi} & -s_{h, \xi}^{*} \\
s_{h, \xi} & c_{h, \xi} Q_{\xi}+Q_{\xi}^{\perp}
\end{array}\right] \in B(\widehat{\mathrm{h}}),
$$


where

$$
s_{h, \xi}:=h^{1 / 2}|\xi\rangle, \quad c_{h, \xi}:=\sqrt{1-s_{h, \xi}^{*} s_{h, \xi}}=\sqrt{1-h\|\xi\|^{2}} \quad \text { and } \quad Q_{\xi}:=P_{\mathbb{C} \xi} .
$$

Then the following hold.

(a) Each $U_{\xi}^{(h)}$ is a unitary operator on $\widehat{\mathrm{h}}$.

(b) The family of *-representations

$$
\widehat{\pi}_{\xi}^{(h)}: \mathrm{A} \rightarrow B(\widehat{\mathrm{h}}), \quad a \mapsto U_{\xi}^{(h) *}(\chi \oplus \pi)(a) U_{\xi}^{(h)} \quad(0<h<h(\xi))
$$

satisfies

$$
\varphi-\Sigma_{h} \circ\left(\widehat{\pi}_{\xi}^{(h)}-\iota_{\widehat{\mathrm{h}}} \circ \chi\right)=\frac{h}{1+c_{h, \xi}} \varphi_{1}-\frac{h^{2}}{\left(1+c_{h, \xi}\right)^{2}} \varphi_{2}
$$

for some completely bounded maps $\varphi_{1}, \varphi_{2}: \mathrm{A} \rightarrow B(\widehat{\mathrm{h}})$ which are independent of $h$. (c) Each *-representation $\widehat{\pi}_{\xi}^{(h)}$ is nondegenerate if (and only if) $\pi$ is.

Proof. For the proof, drop the subscript $\xi$ from $Q, c$ and $s$, and let $0<h<h(\xi)$. Thus

$$
Q=Q_{\xi}, \quad c_{h}=c_{h, \xi}, \quad s_{h}=s_{h, \xi} \quad \text { and } \quad d_{h}:=c_{h}-1 \in[0,1] .
$$

(a) This is evident from the identities

$$
c_{h}^{*}=c_{h}, \quad c_{h}^{2}+s_{h}^{*} s_{h}=1, \quad s_{h}^{*} Q^{\perp}=0 \quad \text { and } \quad s_{h} s_{h}^{*}=\left(1-c_{h}^{2}\right) Q .
$$

(b) Set $v=\pi-\iota_{\mathrm{h}} \circ \chi$ so that $\varphi$ has block matrix form (1.2), note the identities

$$
d_{h}=\frac{-h}{1+c_{h}}\|\xi\|^{2}, \quad\|\xi\|^{2} Q=|\xi\rangle\langle\xi|, \quad c_{h} Q+Q^{\perp}=d_{h} Q+I_{\mathrm{h}},
$$

and define the operators $X, Z \in B(\widehat{\mathrm{h}} ; \mathrm{h})$ by

$$
X:=\|\xi\|^{2}\left[\begin{array}{ll}
0 & Q
\end{array}\right] \text { and } Z:=\left[|\xi\rangle I_{\mathrm{h}}\right] .
$$

Then we have

$$
\begin{aligned}
\Sigma_{h} & \left(\widehat{\pi}_{\xi}^{(h)}(a)-\chi(a) I_{\widehat{h}}\right) \\
& =\mathcal{S}_{h} U_{\xi}^{(h) *}\left[\begin{array}{rr}
0 & \\
v(a)
\end{array}\right] U_{\xi}^{(h)} \mathcal{S}_{h} \\
& =\varphi(a)+d_{h}\left[\begin{array}{l}
0 \\
Q
\end{array}\right] v(a) Z+d_{h} Z^{*} v(a)\left[\begin{array}{ll}
0 & Q
\end{array}\right]+d_{h}^{2}\left[\begin{array}{l}
0 \\
Q
\end{array}\right] v(a)\left[\begin{array}{ll}
0 & Q
\end{array}\right] \\
& =\varphi(a)-\frac{h}{1+c_{h}}\left(X^{*} v(a) Z+Z^{*} v(a) X\right)+\frac{h^{2}}{\left(1+c_{h}\right)^{2}} X^{*} v(a) X,
\end{aligned}
$$

so (b) holds with

$$
\varphi_{1}:=Z^{*} v(\cdot) X+X^{*} v(\cdot) Z \text { and } \varphi_{2}:=X^{*} v(\cdot) X .
$$


(c) This is evident from the unitarity of each $U_{\xi}^{(h)}$ and the fact that $\chi$ is a character.

Remarks. (i) Both $U_{\xi}^{(h)}$ and $\widehat{\pi}_{\xi}^{(h)}$ are norm continuous in $h$; they converge to $I_{\hat{\mathrm{h}}}$ and $\chi \oplus \pi$, respectively, as $h \rightarrow 0$.

(ii) Consider the simplest class of $\chi$-structure map, namely $\varphi=0 \oplus v$, where $v=$ $\pi-\iota \mathrm{h} \circ \chi$ for a *homomorphism $\pi: \mathrm{A} \rightarrow B(\mathrm{~h})$. In this case $\xi=0$ so that $U_{\xi}^{(h)}=I$ and

$$
\widehat{\pi}_{\xi}^{(h)}=\chi \oplus \pi=\varphi+\imath \hat{\mathrm{h}} \quad(h>0) .
$$

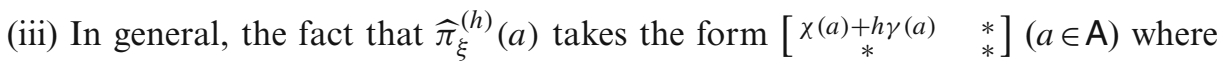
$\gamma:=\omega_{\xi} \circ \nu$, reveals the vector-state realisation

$$
\omega_{e(0)} \circ \widehat{\pi}_{\xi}^{(h)}=\omega_{e(h, \xi)} \circ(\chi \oplus \pi)
$$

for the state

$$
\chi+h \gamma=\left(1-h\|\xi\|^{2}\right) \chi+h\|\xi\|^{2} \omega_{\xi^{\prime}} \circ \pi \quad(0<h<h(\xi)),
$$

where $e(0):=\left(\begin{array}{l}1 \\ 0\end{array}\right) \in \widehat{\mathrm{h}}$,

$$
e(h, \xi):=U_{\xi}^{(h)} e(0)=\left(\begin{array}{c}
\sqrt{1-h\|\xi\|^{2}} \\
h^{1 / 2} \xi
\end{array}\right) \in \widehat{h} \quad \text { and } \quad \xi^{\prime}:= \begin{cases}\|\xi\|^{-1} \xi & \text { if } \xi \neq 0 \\
0 & \text { if } \xi=0 .\end{cases}
$$

Indeed, finding such a representation was the strategy of proof in [11].

(iv) This remark will be used in the proof of Theorem 2.4. Suppose that (instead of $\varphi$ being a $\chi$-structure map) there is a nondegenerate representation $\pi: \mathrm{A} \rightarrow$ $B(\mathrm{H})$, vector $\xi \in \mathrm{H}$ and isometry $D \in B(\mathrm{~h} ; \mathrm{H})$ such that $\varphi$ is given by

$$
\widehat{D}^{*} Z^{*} v(\cdot) Z \widehat{D}, \quad \text { where } \widehat{D}:=\operatorname{diag}[1 \quad D], \quad Z:=\left[\begin{array}{ll}
|\xi\rangle I_{\mathrm{H}}
\end{array}\right] \text { and } v:=\pi-\iota_{\mathrm{H}} \circ \chi .
$$

Then replacing the unitaries $U_{\xi}^{(h)}$ by the isometries $V_{\xi, D}^{(h)}:=U_{\xi}^{(h)} \widehat{D} \in B(\widehat{\mathrm{h}} ; \widehat{\mathrm{H}})$ in the above proof yields a family of completely positive preunital maps

$$
V_{\xi, D}^{(h) *}(\chi \oplus \pi)(\cdot) V_{\xi, D}^{(h)}: \mathrm{A} \rightarrow B(\widehat{\mathrm{h}}) \quad(0<h<h(\xi))
$$

satisfying (2.2) with $V_{\xi, D}^{(h)}$ in place of $U_{\xi}^{(h)}$ and $\widehat{D}^{*} \varphi_{i}^{\prime}(\cdot) \widehat{D}$ in place of $\varphi_{i}$, where $\varphi_{i}^{\prime} \in$ $C B(\mathrm{~A} ; B(\mathrm{H}))(i=1,2)$ is given by $(2.3)$ but with $X, Z \in B(\widehat{H} ; \mathrm{H})$ now.

PROPOSITION 2.3. Let $(\mathrm{A}, \chi)$ be a $C^{*}$-algebra with character and let $\varphi \in$ $C B(A ; B(\widehat{k}))$. Suppose that $\bar{\varphi}(1) \leq 0$ and $\varphi$ is expressible in the form

$$
\varphi_{1}-\varphi_{2} \text { where } \varphi_{1} \in C P(\mathrm{~A} ; B(\widehat{\mathrm{k}})) \text { and } \varphi_{2}=\chi(\cdot)\left(\Delta^{\mathrm{QS}}+|\zeta\rangle\langle e(0)|+| e(0)\rangle\langle\zeta|\right) \text {, }
$$


for a vector $\zeta \in \widehat{\mathrm{k}}$, where $e(0):=\left(\begin{array}{l}1 \\ 0\end{array}\right) \in \widehat{\mathrm{k}}$. Then there is a family of completely positive contractions $\left(\phi^{(h)}: \mathrm{A} \rightarrow B(\widehat{\mathrm{k}})\right)_{0<h<H}$ for some $H>0$ such that

$$
\left\|\varphi-\Sigma_{h} \circ\left(\phi^{(h)}-\iota_{\widehat{\mathrm{k}}} \circ \chi\right)\right\|_{\mathrm{cb}} \rightarrow 0 \text { as } h \rightarrow 0 .
$$

Proof. It follows from Proposition 4.3 and Theorem 4.4 of [26], and their proofs, that there is a Hilbert space $h$ containing $k$ and a $\chi$-structure map $\theta: A \rightarrow B(\widehat{h})$ such that $\varphi$ is the compression of $\theta$ to $B(\widehat{\mathrm{k}})$. The family of *-homomorphisms $\left(\widehat{\pi}_{\xi}^{(h)}: \mathrm{A} \rightarrow B(\widehat{\mathrm{k}})\right)_{0<h<h(\xi)}$ defined in Proposition 2.2 satisfies

$$
\left\|\theta-\Sigma_{h} \circ\left(\widehat{\pi}_{\xi}^{(h)}-\iota_{\mathrm{h}} \circ \chi\right)\right\|_{\mathrm{cb}} \rightarrow 0 \quad \text { as } h \rightarrow 0 .
$$

It follows that (2.6) holds for the compressions $\phi^{(h)}$ of $\widehat{\pi}_{\xi}^{(h)}$ to $B(\widehat{\mathrm{k}})$, which are manifestly completely positive and contractive.

Combining the above results we obtain the following discrete approximation theorem for QS convolution cocycles.

THEOREM 2.4. Let $l$ be a Markov-regular, completely positive, contractive quantum stochastic convolution cocycle on a counital $C^{*}$-bialgebra $\mathrm{B}$, and let $\mathrm{k}$ be its noise dimension space. Then the following hold:

(a) There is a family of completely positive contractions $\left(\psi^{(h)}: \mathrm{B} \rightarrow B(\widehat{\mathrm{k}})\right)_{0<h<H}$ for some $H>0$, such that the convolution iterates $\left(\psi_{n}^{(h)}:=\left(\psi^{(h)}\right)^{\star n}\right)_{n \in \mathbb{Z}_{+}}$satisfy

$$
\sup _{t \in[0, T]}\left\|l_{t, \varepsilon}-\Xi_{[t / h], \varepsilon}^{(h)} \circ \psi_{[t / h]}^{(h)}\right\|_{\mathrm{cb}} \rightarrow 0 \text { as } h \rightarrow 0 \quad\left(T \in \mathbb{R}_{+}, \varepsilon \in \mathcal{E}\right) \text {, }
$$

where again $l_{t, \varepsilon}:=l_{t}(\cdot)|\varepsilon\rangle \in C B(\mathrm{~B} ;|\mathcal{F}\rangle)$ and $\Xi_{[t / h], \varepsilon}^{(h)}$ is given by (2.1).

(b) If $l$ is *-homomorphic, andlor preunital, then each $\psi^{(h)}$ may be chosen to be so too.

Proof. By Theorem 5.2 (a) of [19], we know that $l=l^{\varphi}$ for some map $\varphi \in$ $C B(\mathrm{~B} ; B(\widehat{\mathrm{k}}))$ which has a decomposition of the form $(2.5)$, with $\chi=\epsilon$. The first part therefore follows from Proposition 2.3 and Theorem 2.1. If $l$ is preunital then $\varphi$ may be expressed in the form (2.4) and so, by the remark containing (2.4), it follows that the completely positive maps $\psi^{(h)}$ may be chosen to be preunital.

Now suppose that $l$ is *-homomorphic. Then, by Theorem 5.2 (c) of [19], $\varphi$ is an $\epsilon$-structure map and so, by Theorem $1.1, \varphi$ has an implementing pair $(\pi, \xi)$ with $\pi$ nondegenerate if $l$ is. It therefore follows from Proposition 2.2 that the maps $\psi^{(h)}$ may be chosen to be *homomorphic - and also nondegenerate if the cocycle $l$ is nondegenerate. This completes the proof.

We conclude by restating part of this result in the language of quantum Lévy processes. 
COROLLARY 2.5. Every Markov-regular quantum Lévy process on a multiplier $C^{*}$-bialgebra is a limit, in the pointwise-strong operator topology, of a sequence of quantum random walks.

Proof. This follows from Theorem 2.4 since every Markov-regular quantum Lévy process is equivalent to a Fock space quantum Lévy process, by Corollary 6.2 of [19].

\section{Acknowledgements}

AGS was partially supported by the National Science Center (NCN), Grant no. 2011/01/B/ST1/05011.

Open Access This article is distributed under the terms of the Creative Commons Attribution License which permits any use, distribution, and reproduction in any medium, provided the original author(s) and the source are credited.

\section{References}

1. Applebaum, D., Bhat, B.V.R., Kustermans, J., Lindsay, J.M.: Quantum independent increment processes. In: Franz, U., Schürmann, M. (eds.) From Classical Probability to Quantum Stochastics, vol. I. Lecture Notes in Mathematics, vol. 1865, Springer, Heidelberg (2005)

2. Attal, S., Pautrat, Y.: From repeated to continuous quantum interactions. Ann. Henri Poincaré 7(1), 59-104 (2006)

3. Barndorff-Nielsen, O.E., Franz, U., Gohm, R., Kümmerer, B., Thorbjørnsen, S.: Quantum independent increment processes. In: Franz, U., Schürmann, M. (eds.) Structure of Quantum Lévy Processes, Classical Probability and Physics, vol. II. Lecture Notes in Mathematics, vol. 1866, Springer, Heidelberg (2006)

4. Bédos, E., Tuset, L.: Amenability and co-amenability for locally compact quantum groups. Int. J. Math. 14, 865-884 (2003)

5. Belton, A.C.R.: Approximation via toy Fock space - the vacuum-adapted viewpoint. In: Quantum Stochastics and Information, pp. 3-22. World Scientific, Singapore (2008)

6. Belton, A.C.R.: Random-walk approximation to vacuum cocycles. J. Lond. Math. Soc. (2) 81, 412-434 (2010)

7. Biane, Ph.: Quantum random walk on the dual of SU(n). Probab. Theory Relat. Fields 89(1), 117-129 (1991)

8. Bouten, L., van Handel, R.: Discrete approximation of quantum stochastic models. J. Math. Phys. 49(10), 102109 (2008)

9. Effros, E.G., Ruan, Z.-J.: Operator Spaces. OUP, Oxford (2000)

10. Franz, U., Gohm, R.: Random walks on finite quantum groups. In: [3]

11. Franz, U., Skalski, A.G.: Approximation of quantum Lévy processes by quantum random walks. Proc. Indian Acad. Sci. (Math. Sci.) 118(2), 281-288 (2008)

12. Goswami, D., Sahu, L.: Quantum random walks and vanishing of the second Hochschild cohomology. Lett. Math. Phys. 84(1), 1-14 (2008)

13. Kustermans, J.: Locally compact quantum groups in the universal setting. Int. J. Math. 12(3), 289-338 (2001) 
14. Lance, E.C.: Hilbert $C^{*}$-Modules, a Toolkit for Operator Algebraists. LMS Lecture Note Series, vol. 210. CUP, Cambridge (1995)

15. Lindsay, J.M.: Quantum stochastic analysis - an introduction. In: [1]

16. Lindsay, J.M., Parthasarathy, K.R.: The passage from random walk to diffusion in quantum probability II. Sankhyā Ser. A 50(2), 151-170 (1988)

17. Lindsay, J.M., Skalski, A.G.: Quantum stochastic convolution cocycles. Ann. Inst. Henri Poincaré (B) 41(3), 581-604 (2005)

18. Lindsay, J.M., Skalski, A.G.: Quantum stochastic convolution cocycles II. Commun. Math. Phys. 280(3), 575-610 (2008)

19. Lindsay, J.M., Skalski, A.G.: Quantum stochastic convolution cocycles III. Math. Ann. 352(1), 39-67 (2012)

20. Lindsay, J.M., Wills, S.J.: Existence of Feller cocycles on a $C^{*}$-algebra. Bull. Lond. Math. Soc. 33, 613-621 (2001)

21. Majid, S.: Foundations of Quantum Group Theory. CUP, Cambridge (1995)

22. Neshveyev, S., Tuset, L.: The Martin boundary of a discrete quantum group. J. Reine Angew. Math. 568, 23-70 (2004)

23. Parthasarathy, K.R.: An Introduction to Quantum Stochastic Calculus. Birkhäuser, Basel (1992)

24. Sahu, L.: Quantum random walks and their convergence to Evans-Hudson flows. Proc. Indian Acad. Sci. Math. Sci. 118(3), 443-465 (2008)

25. Schürmann, M.: White Noise on Bialgebras. Lecture Notes in Mathematics, vol. 1544. Springer, Heidelberg (1993)

26. Skalski, A.G.: Completely positive quantum stochastic convolution cocycles and their dilations. Math. Proc. Camb. Philos. Soc. 143(1), 201-219 (2007) 